Portland State University

PDXScholar

Systems Science Faculty Publications and

Presentations

Systems Science

2008

\title{
Emergence of Networks in Distance-Constrained Trade
}

Kumar Venkat

Surya Technologies, Inc.

Wayne W. Wakeland

Portland State University, wakeland@pdx.edu

Follow this and additional works at: https://pdxscholar.library.pdx.edu/sysc_fac

Part of the Systems Engineering Commons

Let us know how access to this document benefits you.

\section{Citation Details}

Venkat K., Wakeland W. (2010) Emergence of Networks in Distance-Constrained Trade. In: Minai A., Braha D., Bar-Yam Y. (eds) Unifying Themes in Complex Systems. Springer, Berlin, Heidelberg

This Pre-Print is brought to you for free and open access. It has been accepted for inclusion in Systems Science Faculty Publications and Presentations by an authorized administrator of PDXScholar. Please contact us if we can make this document more accessible: pdxscholar@pdx.edu. 


\title{
Emergence of Networks in Distance-Constrained Trade
}

\author{
Kumar Venkat \\ Surya Technologies, Inc. \\ 4888 NW Bethany Blvd., Suite K5, \#191 \\ Portland, OR 97229, USA \\ kvenkat@suryatech.com \\ Wayne Wakeland \\ Systems Science Ph.D. Program \\ Portland State University \\ Portland, OR 97207, USA
}

\begin{abstract}
Long-distance trade has been rapidly increasing in recent years. As traders from around the world exchange goods, they form networks with traders as nodes and transactions as links. We use an agent-based model of a simple artificial economy to examine the emergence of trade networks when the distance between traders matters. Distance can become an issue if fuel for transportation becomes expensive or if greenhouse gas emissions from transportation become a major concern. We model the distance constraint as a transaction cost proportional to the amount of goods traded and the distance that those goods must be transported. We find that the resulting network topology is a good indicator of the stability and resilience of the economic system. The topology is random when there is no distance constraint. As the transaction cost increases, the topology transitions into a stable scale-free structure with some clustering, and a large fraction of trade occurs within local regions around the network hubs. Under these conditions, the final welfare of the traders decreases only modestly and environmental efficiency increases significantly when each region has a diverse combination of tradable goods.
\end{abstract}

\subsection{Introduction}

Long-distance trade is an integral part of globalization and has been rapidly increasing in recent years. As traders from around the world exchange goods, they form networks where the traders represent nodes and transactions between them represent links. We examine the emergence of these trade networks using an agent-based model of a simple artificial economy, in which the distance between traders significantly influences the cost of trading. Distance can become an issue if fuel for transportation becomes 
expensive, or if greenhouse gas emissions from the fast-growing transportation sector become a major concern [Venkat 2003]. While information technology is rapidly removing many long-standing obstacles to free trade, the ultimate constraint to trade may well be our ability to physically move material goods between traders over long distances at an acceptable real cost.

We hypothesize that a distance constraint might lead to a restructuring of the fastgrowing society of global traders, and stimulate new kinds of trade relationships and networks. We test our hypothesis in this study using the techniques of agent-based computational economics [Tesfatsion 2006] in a simple setting as a first step. While other studies have focused on the effects of fixed network structures [Wilhite 2001; Wilhite 2006], we take the view that trade networks are highly malleable and arise from the same constraints that influence economic performance. Given the evidence so far that complex systems encode their organizing principles at some level in their topology [Barabasi, et al, 2004], we investigate the evolution and structure of the networks in order to characterize the organization and functioning of our artificial economy.

We model the distance constraint as a transaction cost. This cost reflects some degree of internalization of the real environmental costs of long-distance trade, including fossil fuel depletion and greenhouse gas emissions. We study the effect of this transaction cost under two different initial allocations of tradable goods, one where there are regional differences and the other where the goods are uniformly distributed throughout the world. We are particularly interested in the properties of trade networks that emerge as we vary both the transaction cost and the initial allocation, and we examine how the network properties correlate with economic performance and environmental efficiency.

\subsection{Trade Model}

We formulate the trade problem based on our previous work [Venkat and Wakeland 2006], adapting a simple barter economy that has been used to study economic activity on fixed networks [Wilhite 2001; Wilhite 2006]. Our artificial world consists of 1024 traders spaced uniformly in the four quadrants of a flat space, as shown in Figure 1(a). Each trader is an agent who remains at a fixed location, and is able to trade with others who may be at other arbitrary locations. Traders are presumed to find potential trade partners and negotiate the terms of trade through mechanisms that are independent of their locations, such as globally-accessible electronic trade exchanges.

Each trader starts out with an initial endowment of two durable goods, g1 and g2, ranging from 0 to 1500 units each. The two goods suffer no degradation over time and serve as assets that can be exchanged. There is no production and the aggregate stock of goods changes only to account for the transaction cost as described later. The initial allocation can follow two distinct scenarios, maintaining nearly equal amounts of g1 and g2 in our artificial world:

- "Globally mixed random" (GMR): There are no regional differences. Each trader gets random quantities of the two goods such that the total quantity of both goods together is exactly 1500 units.

- "Local comparative advantage random" (LCAR): The eastern half of the world has more g1 than g2, and the western half has more g2 than g1. Each trader in the east receives at least 1200 units of g1 and no more than 300 units of g2. 
Each trader in the west receives at least 1200 units of g2 and no more than 300 units of g1. The actual amounts are allocated randomly such that each trader starts with a total quantity of 1500 units.

Each trader attempts to maximize the same symmetric Cobb-Douglas welfare function, $\mathrm{U}=\mathrm{g} 1 * \mathrm{~g} 2$. Trade is organized in the form of trade rounds. In each round of trade, traders are chosen in random order and each trader is given a chance to initiate four consecutive trades. The trader then searches the world and finds the best possible trade partners for the four trades. Two traders consummate a trade if their marginal rates of substitutions are different, and if the welfare functions of both traders increase as a result. Trade price between agent $i$ and agent $k$ is determined by the following rule: Price $=\left(\mathrm{g} 2_{\mathrm{i}}+\mathrm{g} 2_{\mathrm{k}}\right) /\left(\mathrm{g} 1_{\mathrm{i}}+\mathrm{g} 1_{\mathrm{k}}\right)$. In each trade, the initiating trader buys or sells one unit of g1 in exchange for an appropriate quantity of g2. Successive trade rounds proceed in this fashion and finally terminate when there are no further profitable trading opportunities.

Each trade incurs a transaction cost computed as: Total transaction cost $=$ distance * quantity of goods bought * unit transaction cost. We vary the unit transaction cost from 0 to 0.25 in our experiments. The total transaction cost is subtracted from the quantity of goods received by each trader in a trade. Traders evaluate this cost in advance and proceed with a trade only if it would still increase their welfare.

\subsection{Results and Discussion}

The trade model was implemented and simulated using NetLogo [Wilensky 1999]. Given the fixed positions of all traders in Figure 1(a), a typical network structure that emerges under the distance-based transaction cost is shown in Figure 1(b). Traders represent nodes of the network and transactions between them represent links. In this section, we probe the origin and structure of these networks and show how they relate to aggregate economic performance in this artificial society.

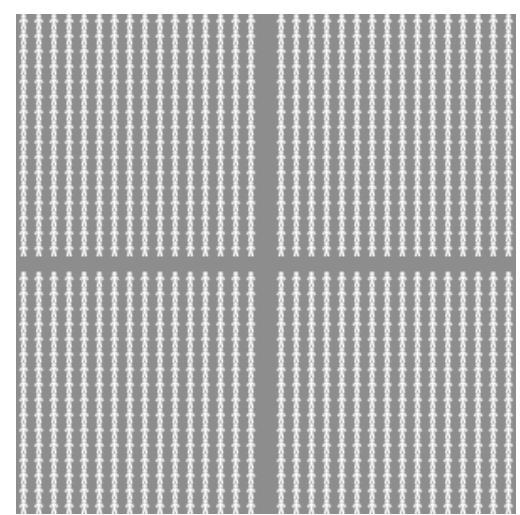

(a)

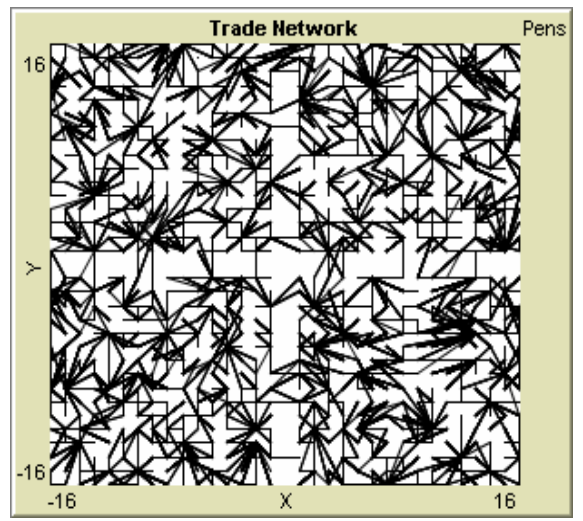

(b)

Figure 1. (a) Location of traders in the artificial world. (b) Typical network structure emerging from distance-constrained trade. 
When the transaction cost is zero, anyone can trade with anyone else in the world. Figure 2(a) shows that both LCAR and GMR produce the same level of final welfare under these conditions. This demonstrates that unconstrained trade can efficiently move goods between traders and achieve a level of welfare that is nearly independent of initial allocations. LCAR does require more trades in order to overcome geographical differences in the initial allocation as seen in Figure 2(b). However, LCAR responds poorly to increases in transaction cost, with welfare dropping to less than 50 percent of the unconstrained case at medium and high costs. The largest drop occurs as the unit transaction cost approaches 0.05 , suggesting a change in the underlying structure, and the welfare characteristic stabilizes at about a cost of 0.1 . In contrast, the average final welfare in the GMR case is within 15 percent of the unconstrained case for all transaction costs.

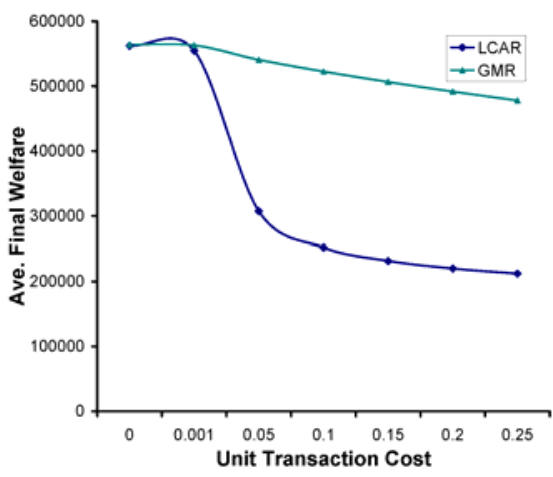

(a)

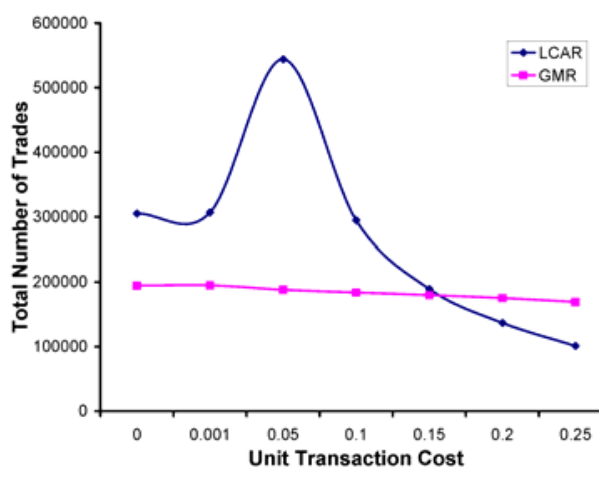

(b)

Figure 2. (a) Average final welfare and (b) total number of trades as functions of unit transaction cost.

As the distance-based transaction cost increases, the average trade distance drops sharply, as seen in Figure 3(a). The trade distance stabilizes at a very low cost in the GMR case, whereas this occurs at a higher cost for LCAR, suggesting that changes in network topology may be occurring at different unit transaction costs in the two cases. Assuming that the greenhouse gas (GHG) emissions produced by each trade are proportional to the quantity of goods and the shipping distance, Figure 3(b) shows the environmental efficiency of the two initial allocations. Clearly, the transaction cost is effective in dramatically increasing the average welfare per unit of greenhouse gas emissions. GMR performs much better than LCAR because the average welfare and the number of trades are very stable while there is a large reduction in the trade distance. The environmental efficiency is relatively stable in this case beyond a cost of 0.05 .

We now examine the network structure in more detail. As seen in Figure 4, the reduction in average network degree closely follows the distance characteristics. The average degree in the GMR case is fairly stable for unit transaction costs between 0.05 and 0.25 . The average degree for LCAR goes through significant changes until a cost of 0.1 , and then continues to change at a slower rate. Stability of the network structure, as measured by the average degree, correlates strongly with relative stability in trade distance, number of trades, welfare and environmental efficiency for both GMR and 
LCAR as the transaction cost is varied. The performance of the GMR case is significantly more stable than LCAR and less sensitive to changes in transaction cost.

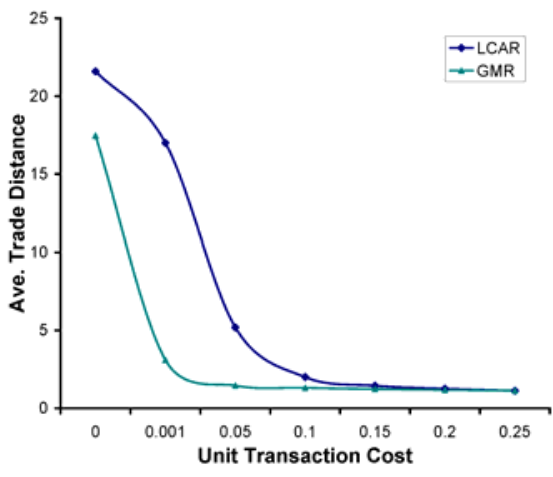

(a)

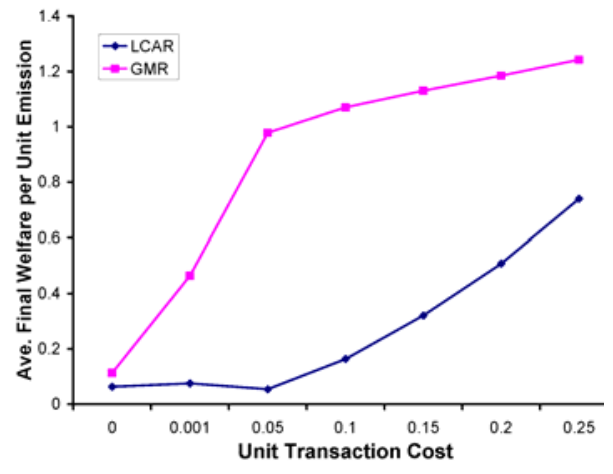

(b)

Figure 3. (a) Average trade distance and (b) environmental efficiency of welfare as functions of unit transaction cost.

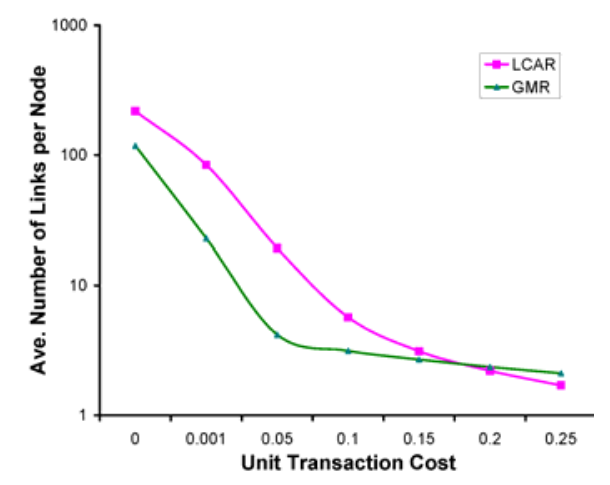

Figure 4. Average network degree as a function of unit transaction cost.

Figure 5 shows the degree distribution for zero and a low unit transaction cost for both GMR and LCAR. Without a transaction cost, the network is clearly random, with a high average degree as also seen in Figure 4. At a low unit transaction cost, the network still remains random, but the average degree is now much smaller since many potential longer-distance links have been eliminated by the transaction cost.

Figure 6 shows the degree distribution for medium and high unit transaction costs. The GMR network displays some scale-free characteristics [Barabasi and Albert 1999] at costs of 0.05 and higher. Most nodes have a small degree while a few hubs have noticeably larger degrees, but the characteristic is limited by the small network size. It is also not an ideal scale-free model because the preferential attachment function [Barabasi, et al, 2004] is highly nonlinear due to the distance constraint. More than 70 percent of the nodes have at least two connections, suggesting some degree of clustering in the neighborhoods around the hubs. In the LCAR scenario, the network is decidedly random at a cost of 0.05 , and moves closer to a scale-free structure at higher 
costs. Note that the formation of scale-free networks corresponds to a regime where trade is generally less sensitive to changes in transaction cost.

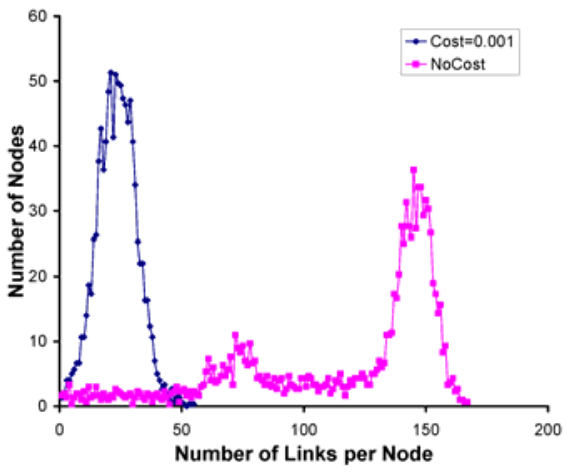

(a)

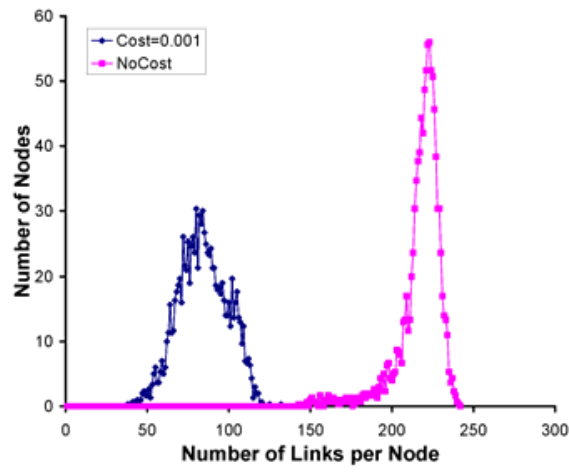

(b)

Figure 5. Degree distribution for zero and low unit transaction costs: (a) GMR. (b) LCAR.

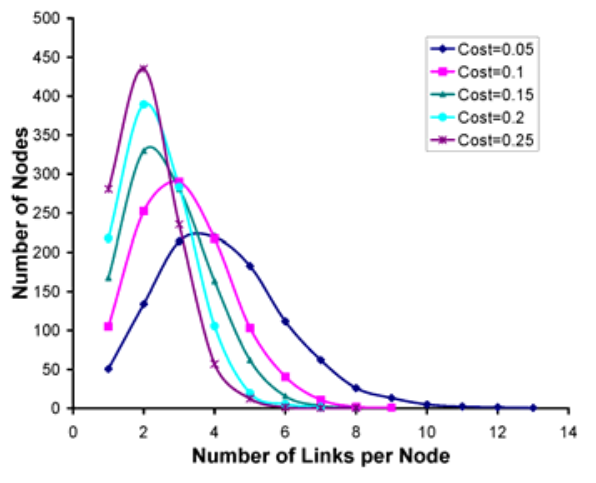

(a)

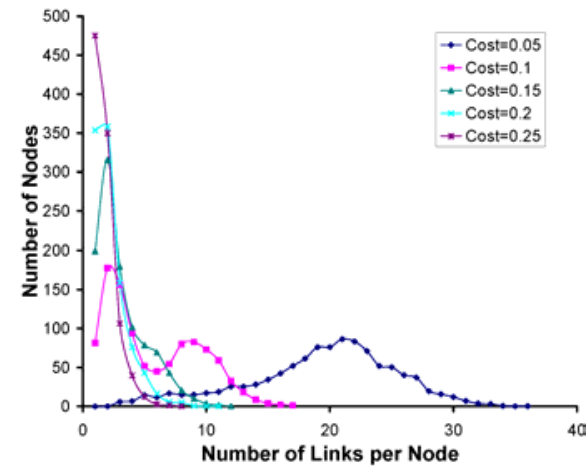

(b)

Figure 6. Degree distribution for medium and high unit transaction costs: (a) GMR. (b) LCAR.

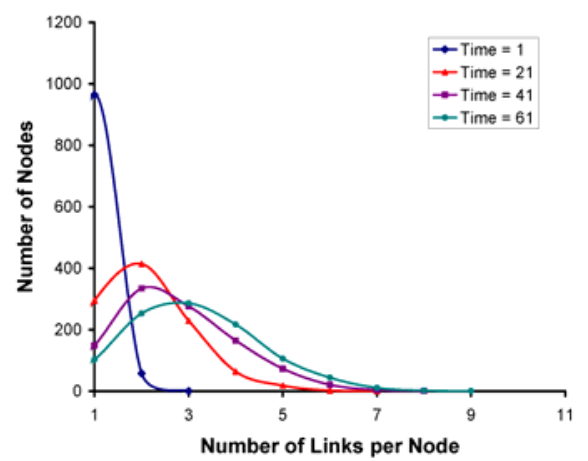

Figure 7. Evolution of the network over time (GMR, Cost $=0.1)$. 
Figure 7 shows the evolution of the network over time in the GMR case, where time steps correspond to trade rounds. The formation of hubs, corresponding to the powerlaw tail in the degree distribution, is reinforced as trade proceeds and is nearly complete after a sufficient number of trade rounds. Once all of the traders have been added to the network, the network stops growing although new connections may still be formed.

In the case of well-known scale-free networks such as the World Wide Web, new nodes link with higher probability to existing nodes that have a large number of connections. In our trade model, the organizing principle turns out to be quite different but still purposeful. The nodes that end up with the largest number of links generally start with lowest initial welfare and hence the largest motivation to engage in trade in order to improve their individual welfare. This motivation to trade makes them local trading hubs for their neighboring nodes. Some of the traders start with lower initial welfare than others because their randomly allocated initial quantities of g1 and g2 are very unequal.

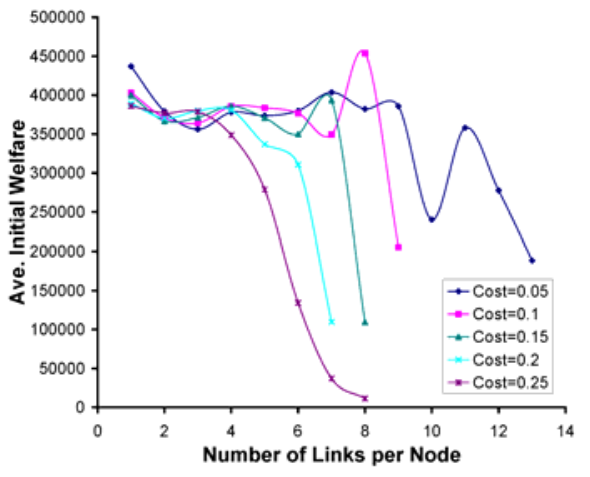

(a)

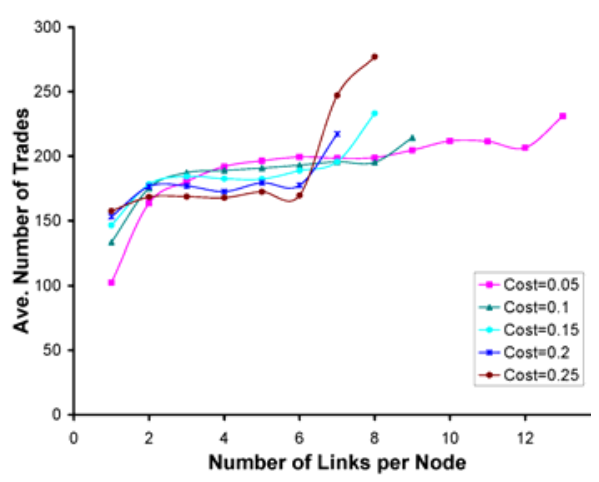

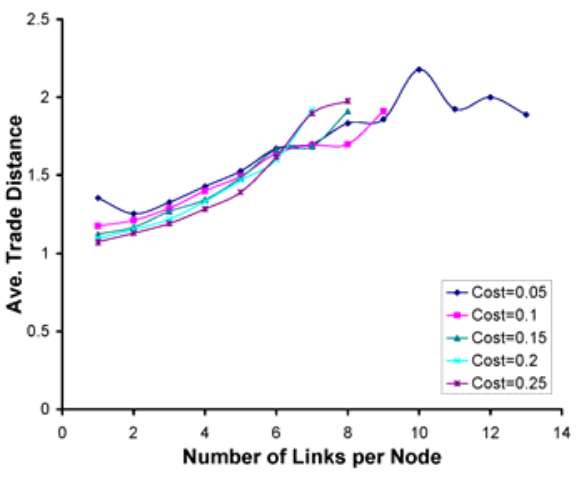

(b)

(c)

Figure 8. Trade characteristics as a function of node degree (GMR): (a) Average initial welfare. (b) Average trade distance. (c) Average number of trades.

Figure 8(a) shows the distribution of initial welfare as a function of the final node degree. Figure 8(b) shows that hubs are likely to trade over longer distances on average, 
driven by their need to increase their welfare. Hubs are also likely to engage in significantly more trade than other nodes, as seen in Figure 8(c).

\subsection{Conclusion}

We have seen that economic performance in the GMR case, as measured by average final welfare, degrades only modestly in response to the transaction cost, while environmental efficiency increases sharply and the number of trades is nearly unchanged. The average degree of the network stabilizes very quickly at a low unit transaction cost and the network gels into a scale-free structure with a certain degree of clustering. In contrast, the response of the LCAR scenario to the transaction cost is much more severe. There is a dramatic decline in welfare coupled with increased number of trades. The network structure remains random until the transaction cost is quite high, at which point it too approaches a scale-free structure. Economic performance stabilizes to some extent when the network takes on scale-free characteristics, but remains considerably worse than the GMR case.

What lessons can we draw from these experiments with a simple artificial world? First, the network topology appears to be a good indicator of the stability and resilience of the economic system. It is a useful way to characterize economic interactions that can provide insights into both organization and function. Second, a distance-based transaction cost - whether imposed by markets or through environmental regulation in the real world - could lead to stable trade networks where most trade occurs within local regions and a small fraction of trade spans longer distances. The hub structure and clustering that emerge in our experiments are ideally suited for local trade. Once such a network has formed, the loss of welfare would be limited if each region has a diverse combination of tradable goods. This suggests that diversified local economies may adapt better to distance constraints than trade regimes where each region specializes in a small number of goods.

\section{References}

Barabasi, A., and Albert, R., 1999, Emergence of Scaling in Random Networks, Science, 286: 509-512.

Barabasi, A., Dezso, Z., Ravasz, E., Yook, S-H., and Oltvai, Z., 2004, Scale-Free and Hierarchical Structures in Complex Networks, Sitges Proceedings on Complex Networks.

Tesfatsion, L, 2006, Agent-Based Computational Economics: A Constructive Approach to Economic Theory, in Handbook of Computational Economics, Vol. 2, edited by L. Tesfatsion and K.L. Judd, North-Holland (Burlington).

Venkat, K., 2003, Global Trade and Climate Change, GreenBiz (www.greenbiz.com).

Venkat, K., and Wakeland, W., 2006, An Agent-Based Model of Trade with Distance-Based Transaction Cost, to appear in Proceedings of the Summer Computer Simulation Conference, The Society for Modeling and Simulation International (San Diego).

Wilensky, U., 1999, NetLogo, Center for Connected Learning and Computer-Based Modeling, Northwestern University (Evanston).

Wilhite, A., 2001, Bilateral Trade and 'Small-World' Networks, Computational Economics, 18: 49-64.

Wilhite, A., 2006, Economic Activity on Fixed Networks, in Handbook of Computational Economics, Vol. 2, edited by L. Tesfatsion and K.L. Judd, North-Holland (Burlington). 